Wei-Guo Duan, Yi-Peng Guo, Long Lin* and Wei-Na Wu

\title{
Crystal structure of bis-( $N^{\prime}$-(5-ethoxycarbonyl-3,4-dimethyl-pyrrol-2-yl- methylidene)-3-hydroxybenzohydrazide- $\left.\mathrm{K}^{2} O, N\right)$ copper(II) - dimethylformamide (1/2), $\mathrm{C}_{40} \mathrm{H}_{50} \mathrm{~N}_{8} \mathrm{O}_{10} \mathrm{Cu}$
}
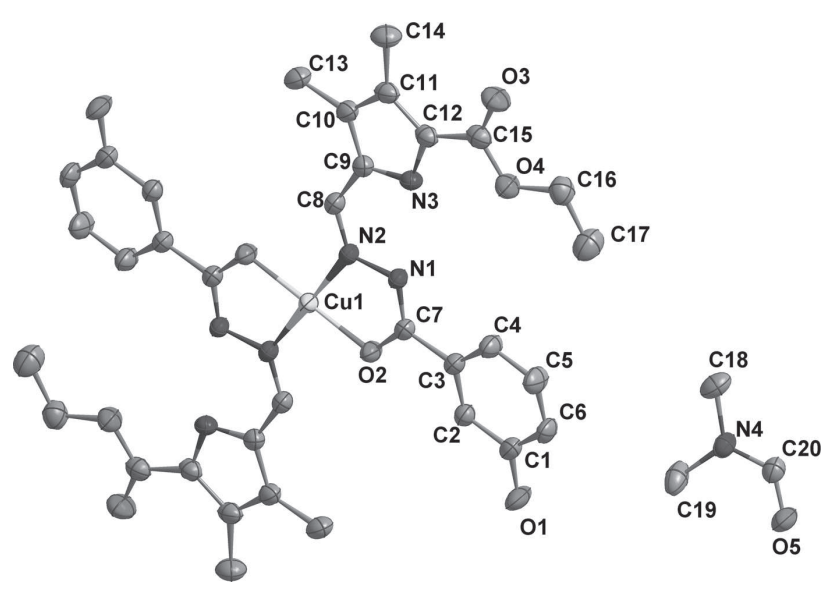

https://doi.org/10.1515/ncrs-2018-0018

Received January 9, 2018; accepted April 30, 2018; available online May 22, 2018

\section{Abstract}

$\mathrm{C}_{40} \mathrm{H}_{50} \mathrm{~N}_{8} \mathrm{O}_{10} \mathrm{Cu}$, triclinic, $P \overline{1} \quad$ (no. 2), $a=6.8237$ (9) $\AA$, $b=12.9229(17) \AA, \quad c=13.3275(18) \AA, \quad \alpha=104.949(2)^{\circ}$, $\beta=104.049(2)^{\circ}, \quad \gamma=99.538(2)^{\circ}, \quad V=1068.3(2) \AA^{3}, \quad Z=1$, $R_{\mathrm{gt}}(F)=0.0497, w R_{\text {ref }}\left(F^{2}\right)=0.1343, T=296(2) \mathrm{K}$.

\section{CCDC no.: 1840534}

The crystal structure is shown in the figure. The asymmetric unit is labelled. Tables 1 and 2 contain details on crystal structure and measurement conditions and a list of the atoms including atomic coordinates and displacement parameters.

\section{Source of material}

The hydrazone ligand and the title complex were prepared according to the literature method [3].

*Corresponding author: Long Lin, School of Materials Science and Engineering, Henan Polytechnic University, Jiaozuo 454000, P.R. China, e-mail: linlong@hpu.edu.cn

Wei-Guo Duan: School of Mathematics and Physics, Weinan Normal University, Weinan 714099, P.R. China

Yi-Peng Guo: School of Materials Science and Engineering, Henan Polytechnic University, Jiaozuo 454000, P.R. China

Wei-Na Wu: College of Chemistry and Chemical Engineering, Henan Polytechnic University, Jiaozuo 454000, P.R. China
Table 1: Data collection and handling.

\begin{tabular}{ll}
\hline Crystal: & Brown block \\
Size: & $0.23 \times 0.20 \times 0.19 \mathrm{~mm}$ \\
Wavelength: & Mo $K \alpha$ radiation $(0.71073 \AA$ A $)$ \\
$\mu:$ & $0.58 \mathrm{~mm}^{-1}$ \\
Diffractometer, scan mode: & APEX Detector, $\varphi$ and $\omega$ \\
$\theta_{\text {max }}$, completeness: & $25.0^{\circ},>99 \%$ \\
$N(h k l)_{\text {measured }}, N(h k l)_{\text {unique }}, R_{\text {int }}:$ & $5702,3747,0.021$ \\
Criterion for $I_{\text {obs }}, N(h k l)_{\mathrm{gt}}:$ & $I_{\text {obs }}>2 \sigma\left(I_{\text {obs }}\right), 2778$ \\
$N(\text { param })_{\text {refined }}:$ & 268 \\
Programs: & SHELX [5], Bruker [6] \\
\hline
\end{tabular}

Table 2: Fractional atomic coordinates and isotropic or equivalent isotropic displacement parameters $\left(\AA^{2}\right)$.

\begin{tabular}{lrrrr}
\hline Atom & $\boldsymbol{x}$ & $\boldsymbol{y}$ & $\boldsymbol{z}$ & $\boldsymbol{U}_{\text {iso }} / \boldsymbol{U}_{\text {eq }}$ \\
\hline Cu1 & 1.000000 & 0.500000 & 0.000000 & $0.0476(2)$ \\
O1 & $0.8905(5)$ & $0.1187(2)$ & $0.2604(2)$ & $0.0776(8)$ \\
H1B & 0.849800 & 0.084257 & 0.298261 & $0.116^{*}$ \\
O2 & $0.9427(4)$ & $0.40078(18)$ & $0.07822(18)$ & $0.0530(6)$ \\
O3 & $0.1448(4)$ & $0.7726(2)$ & $0.2918(2)$ & $0.0793(8)$ \\
O4 & $0.2821(4)$ & $0.6268(2)$ & $0.2928(2)$ & $0.0646(7)$ \\
O5 & $0.2396(4)$ & $-0.0059(2)$ & $0.6170(2)$ & $0.0749(8)$ \\
N1 & $0.7235(4)$ & $0.5099(2)$ & $0.1258(2)$ & $0.0473(7)$ \\
N2 & $0.8059(4)$ & $0.5662(2)$ & $0.0629(2)$ & $0.0473(7)$ \\
N3 & $0.5015(4)$ & $0.6659(2)$ & $0.1603(2)$ & $0.0487(7)$ \\
H3A & 0.512050 & 0.607331 & 0.178387 & $0.058^{*}$ \\
N4 & $0.2398(4)$ & $0.1341(2)$ & $0.5467(2)$ & $0.0556(7)$ \\
C1 & $0.7859(6)$ & $0.1982(3)$ & $0.2540(3)$ & $0.0552(9)$ \\
C2 & $0.8399(5)$ & $0.2685(3)$ & $0.1970(3)$ & $0.0501(8)$ \\
H2A & 0.944850 & 0.259578 & 0.164595 & $0.060^{*}$ \\
C3 & $0.7400(5)$ & $0.3517(3)$ & $0.1877(3)$ & $0.0488(8)$ \\
C4 & $0.5812(7)$ & $0.3639(3)$ & $0.2349(3)$ & $0.0698(11)$ \\
H4A & 0.512540 & 0.419565 & 0.229250 & $0.084^{*}$ \\
C5 & $0.5268(8)$ & $0.2932(3)$ & $0.2898(4)$ & $0.0842(14)$ \\
H5B & 0.418140 & 0.300306 & 0.319809 & $0.101^{*}$ \\
C6 & $0.6295(7)$ & $0.2118(3)$ & $0.3017(3)$ & $0.0721(11)$ \\
H6A & 0.593700 & 0.166229 & 0.341575 & $0.086^{*}$ \\
C7 & $0.8059(5)$ & $0.4250(3)$ & $0.1266(2)$ & $0.0450(7)$ \\
C8 & $0.7487(5)$ & $0.6545(3)$ & $0.0534(2)$ & $0.0485(8)$ \\
H8A & 0.808270 & 0.689189 & 0.011151 & $0.058^{*}$ \\
C9 & $0.6072(5)$ & $0.7064(3)$ & $0.0987(3)$ & $0.0491(8)$ \\
C10 & $0.5470(6)$ & $0.8014(3)$ & $0.0872(3)$ & $0.0521(8)$ \\
C11 & $0.4008(6)$ & $0.8178(3)$ & $0.1430(3)$ & $0.0547(9)$ \\
C12 & $0.3758(5)$ & $0.7331(3)$ & $0.1887(3)$ & $0.0508(8)$ \\
C13 & $0.6296(6)$ & $0.8747(3)$ & $0.0283(3)$ & $0.0669(10)$ \\
H13A & 0.562447 & 0.934621 & 0.032360 & $0.10 *^{*}$ \\
& & & &
\end{tabular}


Table 2 (continued)

\begin{tabular}{lrrrr}
\hline Atom & $\boldsymbol{x}$ & $\boldsymbol{y}$ & $\boldsymbol{z}$ & $\boldsymbol{U}_{\text {iso }}{ }^{*} \boldsymbol{U}_{\text {eq }}$ \\
\hline H13B & 0.777124 & 0.903502 & 0.061368 & $0.100^{*}$ \\
H13C & 0.602206 & 0.832719 & -0.046617 & $0.100^{*}$ \\
C14 & $0.2889(7)$ & $0.9089(3)$ & $0.1510(4)$ & $0.0770(12)$ \\
H14A & 0.334958 & 0.956395 & 0.112160 & $0.116^{*}$ \\
H14B & 0.141621 & 0.877769 & 0.119799 & $0.116^{*}$ \\
H14C & 0.318591 & 0.950859 & 0.226060 & $0.116^{*}$ \\
C15 & $0.2543(6)$ & $0.7157(3)$ & $0.2616(3)$ & $0.0585(9)$ \\
C16 & $0.1776(6)$ & $0.6043(4)$ & $0.3703(3)$ & $0.0716(11)$ \\
H16A & 0.027731 & 0.585877 & 0.337727 & $0.086^{*}$ \\
H16B & 0.215738 & 0.668773 & 0.434099 & $0.086^{*}$ \\
C17 & $0.2424(7)$ & $0.5108(4)$ & $0.4011(4)$ & $0.0950(15)$ \\
H17A & 0.175266 & 0.494100 & 0.452480 & $0.142^{*}$ \\
H17B & 0.203503 & 0.447365 & 0.337419 & $0.142^{*}$ \\
H17C & 0.390742 & 0.529922 & 0.433398 & $0.142^{*}$ \\
C18 & $0.1934(7)$ & $0.2401(3)$ & $0.5521(4)$ & $0.0776(12)$ \\
H18A & 0.226998 & 0.263997 & 0.494456 & $0.116^{*}$ \\
H18B & 0.047935 & 0.233755 & 0.544329 & $0.116^{*}$ \\
H18C & 0.274473 & 0.293087 & 0.620992 & $0.116^{*}$ \\
C19 & $0.3166(7)$ & $0.0817(4)$ & $0.4598(4)$ & $0.0892(15)$ \\
H19A & 0.331837 & 0.129083 & 0.415859 & $0.134^{*}$ \\
H19B & 0.449238 & 0.068471 & 0.490112 & $0.134^{*}$ \\
H19C & 0.219781 & 0.012839 & 0.415660 & $0.134^{*}$ \\
C20 & $0.2073(5)$ & $0.0837(3)$ & $0.6171(3)$ & $0.0583(9)$ \\
H20 & 0.155428 & 0.119374 & 0.671227 & $0.070^{*}$ \\
\hline & & & &
\end{tabular}

\section{Experimental details}

The structure was solved by Direct Methods and refined with the SHELX crystallographic software package [5]. The hydrogen atoms were placed at calculated positions and refined as riding atoms with fixed isotropic displacement parameters.

\section{Comment}

Acylhydrazones are an important class of ligands in coordination chemistry and have been found extensive application in different fields [1]. Our previous work show that acylhydrazone ligands bearing pyrrole units and their complexes exhibit considerable antibacterial and antitumor activity [2-4]. As part of this study the title complex was synthesized and characterized by X-ray diffraction.

The asymmetric unit consists of one half of a copper(II) complex and one DMF molecule ( $c f$. the figure). In the title crystal structure, the central copper ion is coordinated with two enolizated acylhydrazone ligands by NO bidentate donor sets, thus giving a distorted planar square coordination geometry. Two neighbouring DMF molecules link with the complex via pairs of $\mathrm{O}-\mathrm{H} \cdots \mathrm{O}$ hydrogen bonds. $\mathrm{N}-\mathrm{H} \cdots \mathrm{O}$ hydrogen bonds are also presented in the crystal. $\mathrm{Cu}-\mathrm{O}$ and $\mathrm{Cu}-\mathrm{N}$ bond lengths are all in the expected ranges [7].

\section{References}

1. Dong, W.-K.; Li, X.-L.; Wang, L.; Zhang, Y.; Ding, Y.-J.: A new application of Salamo-type bisoximes: as a relay-sensor for $\mathrm{Zn}^{2+} / \mathrm{Cu}^{2+}$ and its novel complexes for successive sensing of $\mathrm{H}^{+} / \mathrm{OH}^{-}$. Sens. Actuators, B 229 (2016) 370-378.

2. Han, X.-F.; Cai, H.-X.; Jia, L.; Wu, W.-N.; Zhang, X.; Xu, J.; Zhang, Z.-P.; Wang, Y.: Syntheses, characterizations and antitumor activities of two copper(II) complexes with an acylhydrazone ligand bearing pyrrole unit. Chin. J. Inorg. Chem. 31 (2015) 1453-1459.

3. Ye, X.-P.; Wang, G.-J.; Pan, P.; Zhang, Z.-P.; Wu, W.-N.; Wang, Y.: Syntheses, crystal structures and biological activities of two $\mathrm{Cu}(\mathrm{II})$ complexes with an acylhydrazone ligand bearing pyrrole unit. Chin. J. Inorg. Chem. 30 (2014) 2789-2795.

4. Ye, X.-P.; Zhu, T.-F.; Wu, W.-N.; Ma, T.-L.; Xu, J.; Zhang, Z.-P.; Wang, Y.; Jia, L.: Syntheses, characterizations and biological activities of two $\mathrm{Cu}(\mathrm{II})$ complexes with acylhydrazone ligand bearing pyrrole unit. Inorg. Chem. Commun. 47 (2014) 60-62.

5. Sheldrick, G. M.: A short history of SHELX. Acta Crystallogr. A64 (2008) 112-122.

6. Bruker. SMART and SAINT. Bruker AXS Inc., Madison, WI, USA (2007).

7. Lu, Q.; Le, K. W.: Crystal structure of bis(2,4-dibromo-6-\{(E)[(4fluorobenzyl)imino]methyl\}phenolato- $\left.\mathrm{k}^{2} \mathrm{~N}, \mathrm{O}\right)$ copper(II), $\mathrm{C}_{28} \mathrm{H}_{18} \mathrm{Br}_{4} \mathrm{~F}_{2} \mathrm{~N}_{2} \mathrm{O}_{2} \mathrm{Cu}$. Z. Kristallogr. NCS 231 (2016) 591-592. 